\title{
Interior Kerr Solutions With The Newman-Janis Algorithm Starting With Static Physically Reasonable Space-Times.
}

\author{
Stefano Viaggiu \\ Dipartimento di Matematica, Universitá di Roma "Tor Vergata", \\ Via della Ricerca Scientifica, 1, I-00133 Roma, Italy \\ E-mail: viaggiu@mat.uniroma2.it \\ (or: stefano.viaggiu@ax0rm1.roma1.infn.it)
}

8th June 2018

\begin{abstract}
We present a simple approach for obtaining Kerr interior solutions with the help of the Newman-Janis algorithm (NJA) starting with static spacetimes describing physically sensible interior Schwarzschild solutions. In this context, the Darmois-Israel (DI) junction conditions are analyzed. Starting from the incompressible Schwarzschild solution, a class of Kerr interior solutions is presented, together with a discussion of the slowly rotating limit. The energy conditions are discussed for the solutions so obtained. Finally, the NJA algorithm is applied to the static, anisotropic, conformally flat solutions found by Stewart leading to interior Kerr solutions with oblate spheroidal boundary surfaces.
\end{abstract}

Keywords: Interior Kerr solutions; Newman-Janis; boundary surfaces. PACS numbers: 04.20.-q, 04.20.Jb

\section{Introduction}

The Kerr metric [1] has been one of the most important astrophysics discovery of the last 40 years. In paricular, this solution gives the exact description of all black holes born from a collapsing star. Further, Kerr claimed that the metric describes the exterior gravitational field of a rotating body. However, since the Kerr solution has been discovered, no physically reasonable sources 
have been found. The problem of finding possible Kerr sources is that, in order to obtain a physically sensible mass distribution, many restrictions must be imposed. First of all, the metric must be joined smoothly to the Kerr one on a reasonable surface for a rotating body and the hydrostatics pressure must be zero on such a surface. Moreover, the energy conditions must hold. Finally, the star must be a non-radiating source and in the static limit a reasonable Schwarzschild interior metric must be obtained. Many techniques exist in the literature to build a trial interior solution [2, 3, 4, 5, 6, 7, 8, 9, 10, First of all, the quoted Geroch conjecture that Kerr metric might have no sources other than a black hole must be noticed. However, a proof is still lacking.

In this paper we present a class of interior solutions that match with the Kerr one on an oblate spheroid with the help of NJA [7. Recently [11, 12, it has been proven that anisotropic fluid interior solutions can be obtained with the NJA, after evaluating the DI 9] junction conditions on an oblate spheroid. Although at first a perfect fluid source seemed the most natural choice, an anisotropic source seems now to be the most appropriate. For example Florides 2, 3, has been able to match an anisotropic rotating fluid on an oblate spheroid with the Kerr metric up to a fifth order of a perturbative parameter. Thus, although a perfect fluid source cannot be excluded a priori, there seem to be no convincing reasons to believe that one must exist.

In [11] the authors present a trial solution requiring that in the static limit, a physically reasonable Schwarzschild interior metric is obtained. To this purpose, the Volkov Oppenheimer [13] equation must be solved because, in order to apply the NJA, we need an explicit seed static metric. However, generally, only numerical methods are available to solve such an equation. In this paper we give a simple constructive approach to obtain a class of interior solutions that could act as physically reasonable Kerr sources.

In section 1 we give a brief introduction to the NJA . In section 2 we present our simple constructive approach. In particular, we study the DI junction conditions in relation to the chosen seed metric. In section 3 we present a class of interior anisotropic solutions starting from the incompressible perfect fluid Schwarzshild metric. Section 4 is devoted to the study of the slowly rotating limit of the solution so obtained. In section 5 the energy conditions and the regularity are discussed. Finally, in section 6 , starting with a static anisotropic conformally flat solution found in [14, a class of interior solutions is obtained, with the help of the NJA algorithm, with oblate spheroidal boundary surfaces. 


\section{NJA Algorithm and the Choice of Coordinates}

The starting point of the NJA algorithm is the static spherically symmetrical seed metric. In spheroidal coordinates $t, r, \theta, \phi$ the seed metric is

$$
d s^{2}=e^{2 \mu(r)} d r^{2}+r^{2}\left(d \theta^{2}+\sin ^{2} \theta d \phi^{2}\right)-e^{2 \nu(r)} d t^{2} .
$$

This metric can be expressed in terms of a null tetrad $l_{\alpha}, n_{\alpha}, m_{\alpha}, \bar{m}_{\alpha}$. To make it short (for more details see [7, 11]), the NJA method consists in taking a complex transformation of the metric (11), once an appropriate coordinate system has been choosen. If Eddington-Finkelstein coordinates are used with $d t=d u-e^{\mu-\nu} d r$, these transformations become

$$
u^{\prime}=u-\imath a \cos \theta, r^{\prime}=r+\imath a \cos \theta, \theta^{\prime}=\theta, \phi^{\prime}=\phi .
$$

In this way, the initial null tetrad relative to (11) acquires complex components dependent on the constant $a$ (rotational parameter). In [11 the authors have been able to cast the metric in a simple form, with only a component $g_{t \phi}$ out of diagonal, compatible with the Kerr metric written in Boyer-Lindquist (BL) coordinates [15. Finally, in BL coordinates, the metric (11) becomes, after the application of the NJA,

$$
\begin{aligned}
& g_{t t}=-e^{2 \nu(r, \theta)}, g_{r r}=\frac{\Sigma}{\sum e^{-2 \mu(r, \theta)}+a^{2} \sin ^{2} \theta}, g_{\theta \theta}=\Sigma, \\
& g_{\phi \phi}=\sin ^{2} \theta\left[\Sigma+a^{2} \sin ^{2} \theta e^{\nu(r, \theta)}\left(2 e^{\mu(r, \theta)}-e^{\nu(r, \theta)}\right)\right], \\
& g_{t \phi}=a e^{\nu(r, \theta)} \sin ^{2} \theta\left(e^{\mu(r, \theta)}-e^{\nu(r, \theta)}\right),
\end{aligned}
$$

where $\Sigma=r^{2}+a^{2} \cos ^{2} \theta$ and $\nu, \mu$ now depend on $r, \theta$. Setting

$$
e^{2 \mu}=e^{-2 \nu}=\frac{r^{2}+a^{2} \cos ^{2} \theta}{r^{2}-2 r m+a^{2} \cos ^{2} \theta},
$$

we obtain the Kerr solution. This can be obtained, in Cartesian coordinates $(t, x, y, z)$, in the Kerr-Shild form:

$$
\begin{aligned}
& d s^{2}=d x^{2}+d y^{2}+d z^{2}-d t^{2} \\
& +\frac{2 m}{r^{4}+a^{2} z^{2}}\left[\frac{r(x d x+y d y)+a(x d y-y d x)}{r^{2}+a^{2}}+\frac{z}{r} d z+d t\right]^{2},
\end{aligned}
$$

where the variable $r$ is defined as:

$$
\frac{x^{2}+y^{2}}{r^{2}+a^{2}}+\frac{z^{2}}{r^{2}}=1
$$


Performing the coordinate transformation given by [15]

$$
\begin{aligned}
& x=r \sin \theta \cos \phi+a \sin \theta \sin \phi \\
& y=r \sin \theta \sin \phi-a \sin \theta \cos \phi, \\
& z=r \cos \theta, \quad t \rightarrow t-2 m \int \frac{r d r}{r^{2}-2 m r+a^{2}},
\end{aligned}
$$

with the transformations (7), the metric takes exactly expression (3) with (4). From (6) we deduce that surfaces with $r=$ const. are oblate spheroids that in the limit $a=0$ reduce to spheres.

\section{Matching Conditions and Generation of Kerr Interior Solutions from Static Ones}

Given two space-times with Lorentzian signature $(-+++), M^{+}$and $M^{-}$, with related metrics $g_{\alpha \beta}^{+}$and $g_{\alpha \beta}^{-}$(for the space-time (3) we can use the same coordinate system both for $M^{+}$and $M^{-}$), we can construct a new manifold $M$ by joining the two space-times on a non-null 3 -surface $S$, with the help of the junction formalism [9, 10]. To join $M^{+}$and $M^{-}$on $S$, we must calculate the metric tensor $g_{i j}^{ \pm}$on $S$ (first fundamental form) and the extrinsic curvature (second fundamental form) $K_{i j}$. In terms of the unit normal to the surface $n^{\alpha}$ this is given by $K_{i j}=n_{i ; j}$, where ";" denotes the covariant derivative and the equation for $S$ for non-null surfaces is $f\left(x^{\alpha}\right)=0$. Thus $n_{\gamma}= \pm \frac{\partial_{\gamma} f}{\sqrt{\partial_{\beta} f \partial^{\beta} f}}$, the sign \pm depending on the orientation of the 4 vector field $n_{\gamma}$.

The DI conditions for $M^{+}$and $M^{-}$on $S$ are:

$$
\begin{aligned}
& {\left[g_{i j}\right]=g_{i j}^{+}-g_{i j}^{-}=0} \\
& {\left[K_{i j}\right]=K_{i j}^{+}-K_{i j}^{-}=0 .}
\end{aligned}
$$

In what follows the subscript "-" denotes the interior solution.

If both (8) and (9) are satisfied, then $S$ is a boundary surface. If only (8) is satisfied, $S$ is a thin shell surface. If we choose coordinates (17), on an oblate spheroid with $r=R$ ( $R$ a constant) for the line element (3), then the conditions (8) become

$$
\begin{aligned}
& \left(e^{2 \nu_{+}}\right)_{S}=\left(e^{2 \nu_{-}}\right)_{S}, \\
& \left(e^{2 \mu_{+}}\right)_{S}=\left(e^{2 \mu_{-}}\right)_{S},
\end{aligned}
$$


while conditions (9) are

$$
\begin{aligned}
& \left(e^{2 \nu_{+}}{ }_{, r}\right)_{S}=\left(e^{2 \nu_{-}}{ }_{, r}\right)_{S}, \\
& \left(e^{2 \mu_{+}}{ }_{, r}\right)_{S}=\left(e^{2 \mu_{-}}{ }_{, r}\right)_{S},
\end{aligned}
$$

where subindices "," denote the partial derivative.

In terms of the Kerr exterior solution (44), conditions (10) now look:

$$
\begin{aligned}
\left(e^{2 \nu_{-}}\right)_{S} & =\frac{R^{2}+a^{2} \cos ^{2} \theta-2 m R}{\left(R^{2}+a^{2} \cos ^{2} \theta\right)}, \\
\left(e^{2 \mu_{-}}\right)_{S} & =\frac{R^{2}+a^{2} \cos ^{2} \theta}{\left(R^{2}+a^{2} \cos ^{2} \theta-2 m R\right)},
\end{aligned}
$$

and (11)

$$
\begin{aligned}
\left(e^{2 \nu_{-}}\right)_{S} & =\frac{2 m\left(R^{2}-a^{2} \cos ^{2} \theta\right)}{\left(R^{2}+a^{2} \cos ^{2} \theta\right)^{2}}, \\
\left(e^{2 \mu_{-}}{ }_{, r}\right)_{S} & =\frac{2 m\left(a^{2} \cos ^{2} \theta-R^{2}\right)}{\left(R^{2}+a^{2} \cos ^{2} \theta-2 m R\right)^{2}} .
\end{aligned}
$$

Our strategy for finding Kerr interior solutions is the following: instead of starting with a stationary trial solution with conditions (8) (9), we start with a static physically reasonable interior Schwarzschild solution. In this case we only need to know the explicit form of the static line element, but, in order to find the pressure and the density profile of the star, it is not necessary that the Volkov-Oppenheimer equation be solved analitically. Further, it must be ensured that, starting with a trial stationary solution with conditions (8) and (9), the static solution is free of conical singularity: this imposes some additional conditions on the metric components. If a static seed metric is chosen, the next step is to decide if the surface $S$ is a boundary surface or a thin shell surface. Finally, if the static seed metric is composed of elementary functions, we can find the expression (depending on the parameter " $a$ ") that verifies the appropriate junction conditions. In the next section we apply these reasonings to the imcompressible perfect fluid Schwarzschild solution.

\section{Example One: The Incompressible Perfect Fluid Schwarzschild Solution}

In [6], the authors first applied the NJA using as seed metric the incompressible Schwarzschild solution. However, they did not use the transformed metric in the simple form (3), but rather applied the NJA algorithm performing 
a complex transformation on the Schwarzschild interior solution after taking the two constants of this solution as functions of $r$. Consequently, the result they found in the static limit represents an anisotropic fluid solution, not the starting perfect fluid one. Furthermore, this solution cannot satisfy the strong energy condition.

The incompressible Schwarzschild solution is given by (1) with

$$
\begin{aligned}
e^{2 \mu} & =\frac{R^{3}}{R^{3}-2 m r^{2}}, \\
e^{2 \nu} & =\left[\frac{3}{2} \sqrt{1-\frac{2 m}{R}}-\frac{1}{2} \sqrt{1-\frac{2 m r^{2}}{R^{3}}}\right]^{2} .
\end{aligned}
$$

We have then (in appropriate units) $G_{\mu \nu}=T_{\mu \nu}$ and therefore for the massenergy density $\epsilon$ and for the radial pressure $p$ we obtain

$$
\begin{aligned}
& \epsilon= \frac{6 m}{R^{3}}, \\
& p=\frac{\epsilon\left(\sqrt{1-\frac{2 m r^{2}}{R^{3}}}-\sqrt{1-\frac{2 m}{R}}\right)}{\left(3 \sqrt{1-\frac{2 m}{R}}-\sqrt{1-\frac{2 m r^{2}}{R^{3}}}\right)} .
\end{aligned}
$$

Solutions (16) and (17) are stable under perturbations when (Bondi limit [16]) $R>\frac{9}{4} m$. The Schwarzschild interior solution is acausal, and thus the dominant energy condition [17] cannot be satisfied for the energy-momentum tensor $T_{\mu \nu}$ of metric (3). Despite this, the unphysical character becomes less important if this solution is considered as a limiting case of the class of solutions with non-increasing outwards density [18. Furthermore, the incompressible Schwarzschild solution is the unique conformally flat, static perfect fluid solution and it can be a model to describe the core of a neutron star.

First of all we must decide what boundary conditions must be imposed to the stationary metric (3) with the seed metric (16) and (17), i.e. if only (8) or both (8) and (9) must be considered.

For (16), (17), the continuity of $\nu_{, r}(r)$ assures the continuity of the hydrostatic pressure $p$ (19). On the contrary, the continuity of $\mu_{, r}$ is not necessary because of the equation:

$$
\epsilon r^{2}=1-e^{-2 \mu(r)}\left[1-2 r \mu_{, r}\right]
$$


In other words, the energy density is constant and thus cannot be vanishing on $r=R$. In fact:

$$
\left(e^{2 \mu_{+}}{ }_{, r}\right)_{S}=\frac{-2 m}{(R-2 m)^{2}} \neq \frac{4 m}{(R-2 m)^{2}}=\left(e^{2 \mu_{-}}\right)_{S} .
$$

However, it must be pointed out that conditions (14) and (15) have been obtained (see [11]) assuming $a \neq 0$. When we derive condition (14)-(15), the parameter $a$ appears as an overall factor in (15), and thus in the limit $a=0$ the only surviving condition for the continuity of $K_{i j}$ is (14).

Conversely, in the stationary case with the line element (3), for the continuity of $K_{i j}$ (boundary surface), both (14) and (15) must be satisfied. Furthermore, if we consider the static limit as an analytic limit of stationary space-times, expression (21) says that condition (15) cannot be satisfied when the parameter $a$ is present. This fact can be confirmed performing the limit $a=0$ in (15), obtaining exactly expression (21). Consequently, for the interior stationary solutions generated by (16) and (17), only conditions (12) and (13) must be imposed, for a thin shell surface.

Moreover, it is possible to impose to the stationary metric (3) the condition (14) (satisfied for the chosen static seed solution ), but it appears to be not necessary. By inspection of (16), it is easy to see that the most general expression for $e^{2 \mu(r, \theta)}$ satisfying condition (13) is:

$$
e^{2 \mu_{-}}=\frac{R^{3}+R a^{2} \cos ^{2} \theta}{R^{3}+R a^{2} \cos ^{2} \theta-2 m r^{2}}+B\left(r, \theta, a^{2}\right),
$$

where $B\left(r, \theta, a^{2}\right)$ is an arbitrary function such that:

$$
B\left(R, \theta, a^{2}\right)=B(r, \theta, 0)=0 .
$$

Obviously, because of the axial simmetry of (3), only even powers in $a$ are allowed, and $B\left(r, \theta, a^{2}\right)$ must be such that expression (22) is non-negative. If $B\left(r, \theta, a^{2}\right)$ is a regular function of its arguments, then expression (22) is regular for $R>2 m$. Finally, the most general expression for $e^{2 \nu(r, \theta)}$ that satisfies condition (12) is:

$$
\begin{aligned}
& e^{2 \nu_{-}}=\frac{5}{2}-\frac{9 m R}{2\left(R^{2}+a^{2} \cos ^{2} \theta\right)}-\frac{m r^{2}}{2 R\left(R^{2}+a^{2} \cos ^{2} \theta\right)} \\
& -\frac{3}{2} \sqrt{1-\frac{2 m R}{\left(R^{2}+a^{2} \cos ^{2} \theta\right)}} \sqrt{1-\frac{2 m R\left(r^{2}+a^{2} \cos ^{2} \theta\right)}{\left(R^{2}+a^{2} \cos ^{2} \theta\right)^{2}}}+H\left(r, \theta, a^{2}\right)(2
\end{aligned}
$$

where $H\left(r, \theta, a^{2}\right)$ is an arbitrary regular function with

$$
H\left(R, \theta, a^{2}\right)=H(r, \theta, 0)=0
$$


and such that expression (24) is non-negative. Even in this case, (24) is regular for $R>2 m$. If we impose condition (14), we have:

$$
\begin{aligned}
& e^{2 \nu_{-}}=H_{\alpha}\left(a^{2}, r, \theta\right)+\left[\frac{5}{2}-\frac{9 m R}{2\left(R^{2}+a^{2} \cos ^{2} \theta\right)}-\frac{m r^{2}}{2 R\left(R^{2}+a^{2} \cos ^{2} \theta\right)}\right. \\
& -\frac{3}{2} \sqrt{1-\frac{2 m R}{\left(R^{2}+a^{2} \cos ^{2} \theta\right)}} \sqrt{\left.1-\frac{2 m R\left(r^{2}+a^{2} \cos ^{2} \theta\right)}{\left(R^{2}+a^{2} \cos ^{2} \theta\right)^{2}}\right] e^{\alpha\left(a^{2}, r, \theta\right)},}
\end{aligned}
$$

where $\alpha$ is a regular function such that $\alpha(0, r, \theta)=\alpha\left(a^{2}, R, \theta\right)=0$. Also $H_{\alpha}$ is a regular vanishing function for $a=0$ and $r=R$ and depends on the choice made for $\alpha$. For example, setting $\alpha=0$, we have:

$$
H_{0}\left(r, \theta, a^{2}\right)=\frac{c_{H} H(r) a^{2} \cos ^{2} \theta}{\left(R^{2}+a^{2} \cos ^{2} \theta\right)^{2}},
$$

where $H(r)$ is a regular function such that $H(R)=0$ and the constant $c_{H}$ in (27) depends on $H(r)$. For example, if $H(r)=\left(R^{2}-r^{2}\right)$ then $c_{H}=\frac{m}{2 R}$. Expressions (22) and (24), when substituted in (3) give our class of stationary interior solutions describing an anisotropic fluid.

In the literature, the only known exact anisotropic fluid solution is given in [19]. In the next section we study the solutions so obtained at the first order in the rotational parameter $a$.

\section{Slowly Rotating Limit}

The suitable metric to describe a slowly rotating star is given by [20]

$$
d s^{2}=e^{2 \mu(r, \theta)} d r^{2}+e^{2 \mu_{2}(r, \theta)} d \theta^{2}+e^{2 \psi(r, \theta)}[d \phi+\omega(r, \theta) d t]^{2}-e^{2 \nu(r, \theta)} d t^{2}
$$

where, with $\Omega=\frac{d \phi}{d t}$ denoting the angular velocity as seen from a rest observer at spatial infinity, we have:

$$
\begin{array}{ll}
\nu=\nu_{0}+o(\Omega) \quad, \quad \psi=\psi_{0}+o(\Omega) \\
\mu=\mu_{0}+o(\Omega) \quad, \quad \mu_{2}=\mu_{20}+o(\Omega) .
\end{array}
$$

Here $\nu_{0}, \psi_{0} \cdots$ represent the static metric functions. By slow rotation we mean that the star rotates with an angular velocity $\Omega$ so small that distorsions to its shape of order $\Omega^{2}$ can be ignored. The metric (28), at order $\Omega$, reduces to:

$$
d s^{2}=e^{2 \mu(r)} d r^{2}+r^{2}\left(d \theta^{2}+\sin ^{2} \theta d \phi^{2}\right)+2 \omega(r) \sin ^{2} \theta d t d \phi-e^{2 \nu(r)} d t^{2},
$$


with $\mu$ and $\nu$ given by (16) and (17) and $\omega(r)=a e^{\nu}\left(e^{\mu}-e^{\nu}\right)$.

Therefore, at the boundary $R$ we have $\omega(R)=\frac{2 m}{R}$, as expected for a slowly rotating star in the vacuum.

Note that since at order $\Omega$ the star is spherical (the distorsion to its spherical shape appears at order $\Omega^{2}$, where the centrifugal force comes in action), we have $\Omega=\frac{5 a}{2 R^{2}}$ (the inertial momentum $I$ of a sphere is $I=\frac{2}{5} m R^{2}$ ). With the metric (30), the only first order component of the Einstein's tensor $G_{\mu \nu}$ is $G_{t \phi}$. The other non-vanishing components are zero order expressions in $a$ and are thus the same of the static metric. Then, the energy-momentum tensor at the order $a$ has the $t t, r r, \theta \theta, \phi \phi$ components of the metric (16) and (17) of a perfect fluid with

$$
\begin{aligned}
& T_{\mu \nu}=(\epsilon+p) u_{\mu} u_{\nu}+p g_{\mu \nu}, \\
& u^{t}=\frac{d t}{d s}=e^{-\nu}, u^{\theta}=u^{r}=0, u^{\phi}=\frac{d \phi}{d s}=\frac{5 a}{2 R^{2}} e^{-\nu} .
\end{aligned}
$$

Conversely, the equation involving $G_{t \phi}$ is

$$
\begin{aligned}
G_{t \phi} & =\sin ^{2} \theta e^{-2 \mu}\left[\frac{\omega}{r^{2}}+\frac{\omega_{, r} \mu_{, r}}{2}+\frac{\omega_{, r} \nu_{, r}}{2}-\frac{2 \omega \mu_{, r}}{r}+\right. \\
& \left.+\omega \nu_{, r}^{2}-\omega \nu_{, r} \mu_{, r}-\frac{\omega_{, r, r}}{2}+\omega \nu_{, r, r}\right]+o(a),
\end{aligned}
$$

Thus, once $\nu$ and $\mu$ are substituted with (16) and (17), we get:

$$
G_{t \phi}=T_{t \phi}=\sin ^{2} \theta\left[-r^{2} \epsilon\left(\frac{\omega}{r^{2}}+\frac{5 a}{2 R^{2}}\right)-\frac{5 a p r^{2}}{2 R^{2}}+\Phi_{t \phi}(r)\right]+o(a) .
$$

If the star, at order $a$, was described by a perfect fluid, then the quantity $\Phi_{t \phi}$ would be identically 0 . This non-zero component makes the fluid a non-perfect one. The energy-momentum tensor in the slowly rotating approximation has the same diagonal components of the static Schwharzschild one with a component $(t \phi)$ out of diagonal composed of a perfect fluid part and anisotropic part given by $\Phi_{t \phi}$ : the energy density $\epsilon$ and the radial pressure $p$ are again given by (18) and (19).

Concerning the energy conditions, for a tensor of the type considered (type I, see [17]), denoting with $\lambda_{0}$ the eigenvalue corresponding to the timelike eigenvector, and with $\lambda_{i}(i=1,2,3)$ the eigenvalues corresponding to spacelike eigenvectors, we have for the weak energy condition:

$$
-\lambda_{0} \geq 0, \quad-\lambda_{0}+\lambda_{i} \geq 0
$$

for the strong energy condition:

$$
-\lambda_{0}+\sum \lambda_{i} \geq 0 \quad, \quad-\lambda_{0}+\lambda_{i} \geq 0
$$


and for the dominant energy condition:

$$
-\lambda_{0} \geq 0 \quad, \quad \lambda_{0} \leq \lambda_{i} \leq-\lambda_{0},
$$

where the eigenvalues are solutions of the equation:

$$
\left|T_{\alpha \beta}-\lambda g_{\alpha \beta}\right|=0 .
$$

It is a simple matter to verify that at order $a$ the equation (37) is the same as the static limit, and thus (34) and (35) are satisfied (remember that the seed static solution considered is acausal). In particular we have:

$$
\lambda_{0}=-\epsilon+o(a) \quad, \quad \lambda_{i}=p(r)+o(a) .
$$

In the next section we collect some remarks on the energy conditions for our solution .

\section{Considerations on Energy Conditions}

The eigenvalue equation (37) for the metric (3), becomes:

$$
\left|\begin{array}{cccc}
T_{t t}-\lambda g_{t t} & 0 & 0 & T_{t \phi}-\lambda g_{t \phi} \\
0 & T_{r r}-\lambda g_{r r} & T_{r \theta} & 0 \\
0 & T_{r \theta} & T_{\theta \theta}-\lambda g_{\theta \theta} & 0 \\
T_{t \phi}-\lambda g_{t \phi} & 0 & 0 & T_{\phi \phi}-\lambda g_{\phi \phi}
\end{array}\right|=0
$$

and the eigenvalues are thus given by:

$$
\begin{aligned}
& \lambda_{0,1}=\frac{P \pm \sqrt{P^{2}-4\left(g_{t t} g_{\phi \phi}-g_{t \phi}^{2}\right)\left(T_{t t} T_{\phi \phi}-T_{t \phi}^{2}\right)}}{2\left[g_{t t} g_{\phi \phi}-g_{t \phi}^{2}\right]} \\
& P=\left(T_{t t} g_{\phi \phi}+g_{t t} T_{\phi \phi}-2 T_{t \phi} g_{t \phi}\right) \\
& \lambda_{2,3}=\frac{Q \pm \sqrt{Q^{2}-4 g_{r r} g_{\theta \theta}\left(T_{r r} T_{\theta \theta}-T_{r \theta}^{2}\right)}}{\left(2 g_{r r} g_{\theta \theta}\right)} \\
& Q=\left(T_{r r} g_{\theta \theta}+g_{r r} T_{\theta \theta}\right) .
\end{aligned}
$$

First of all, note that it is not possible to perform a perturbative expansion with respect to the parameter $a$. In fact, the metric (3) with (22) and (24) is not continuous in the variables $(r, a)$ in all the interior shell. For example, if we take the metric element $g_{r r}$ with (22) and (23), then the limits for 
$a \rightarrow 0, r \rightarrow 0$ cannot be exchanged. Further, if we expand formally the component $g_{r r}$ up to the order $a^{2}$, we get:

$$
\begin{aligned}
& g_{r r}=\frac{R^{3}}{R^{3}-2 m r^{2}}+ \\
& \frac{a^{2}\left[-2 m r^{4} R \cos ^{2} \theta+B\left(r^{2} R^{6}-4 r^{4} R^{3} m+4 r^{6} m^{2}\right)-R^{6} \sin ^{2} \theta\right]}{r^{2}\left(R^{3}-2 m r^{2}\right)^{2}} .
\end{aligned}
$$

If we take $B(r, \theta)$ to be a regular function at $r=0$, then expression (41) is divergent to $-\infty$, whereas the component $g_{r r}$, given by (3) with (22), is bounded in a neighbourhood of $r=0$. Therefore, a perturbative expansion in the parameter " $a$ " is possible only in the slowly rotating limit, where the functions $\mu(r, \theta)$ and $\nu(r, \theta)$ are the static ones.

The study of the behaviour of the metric in $r=0$ is important for the energy conditions since a singularity can arise at such a point. Expressions (39) and (40) show a complicated dependence on $H\left(r, \theta, a^{2}\right), B\left(r, \theta, a^{2}\right)$. Therefore, the study of (34)-(36) is not a simple task. However, some remarks can be made. First of all, the components $T_{t t}, T_{t \phi}, T_{r r}, T_{r \theta}, T_{\theta \theta}$ have a finite limit on the axis at $\theta=0, \pi$, and for $T_{\phi \phi}$ we have $T_{\phi \phi} \sim \sin ^{2} \theta$. Therefore the eigenvalues (39) and (40) are well defined on the axis where the norm of the spacelike Killing vector $\xi_{\phi}$ is vanishing. Further, the metric functions are regular when $R>2 \mathrm{~m}$. The only singularity can arise when the determinant of the metric is vanishing, i.e. at $r=0, \theta=\frac{\pi}{2}$ (ring singularity).

Concerning the energy conditions, the inequality $-\lambda_{0} \geq 0$ is satisfied if (for example) $T_{t t} T_{\phi \phi}-T_{t \phi}^{2} \geq 0$ (with $g_{\phi \phi} \geq 0, g_{t t} \leq 0$ ) and consequently $-\lambda_{0}+\lambda_{1} \geq 0$ is satisfied. The energy conditions cannot be satisfied if $\lambda_{2}, \lambda_{3}$ have a large divergent negative part and $\lambda_{0}$ with a less negative divergent part. The stress-energy tensor has only $(R>2 m)$ a ring singularity at $r=0, \theta=\frac{\pi}{2}$. In a neighbourhood of the ring, the most rapidly diverging part looks as follows (with $\Sigma=r^{2}+a^{2} \cos ^{2} \theta$ ):

$$
\begin{aligned}
& T_{t t} \sim \Sigma^{-4}, T_{t \phi} \sim \Sigma^{-4}, T_{\phi \phi} \sim \Sigma^{-4}, \\
& T_{r r} \sim \Sigma^{-2}, T_{\theta \theta} \sim \Sigma^{-2}, T_{r \theta} \sim \Sigma^{-1} .
\end{aligned}
$$

Therefore, we can find the general expressions needed for $B\left(r, \theta, a^{2}\right), H\left(r, \theta, a^{2}\right)$ such that we can reduce the most rapidly divergent part in (39) and (40). We can take:

$$
\begin{aligned}
& B\left(r, \theta, a^{2}\right)=\Sigma^{k}\left[A\left(r, \theta, a^{2}\right)(R-r)^{\epsilon}+L\left(r, \theta, a^{2}\right)\left(R^{\gamma}-r^{\gamma}\right)\right]+ \\
& C\left(r, \theta, a^{2}\right)(R-r)^{\delta}+D\left(r, \theta, a^{2}\right)\left(R^{\Delta}-r^{\Delta}\right)+ \\
& {\left[\Sigma^{\psi}-\left(R^{2}+a^{2} \cos ^{2} \theta\right)^{\psi}\right]^{\beta} E\left(r, \theta, a^{2}\right),}
\end{aligned}
$$


where $(\epsilon, \gamma, \delta, \Delta, \psi, \beta)$ are non-negative constants (assuming the regularity of the metric coefficients) and the functions $A, L, C, D, E$ are regular functions of their arguments and vanish for $a=0$. A similar expression follows for $H\left(r, \theta, a^{2}\right)$ with obviously different constants $\left(\epsilon^{\prime}, \gamma^{\prime} \cdots\right)$ and functions $A^{\prime}, L^{\prime} \cdots$. The next step is to choose the parameters and functions in (43) in such a way that $-\lambda_{0} \geq 0$ and that $\lambda_{0}$ has a divergent part at most of order $\sim \Sigma^{-1}$ : this way the value of the volume integral of $\lambda_{0}$ on $r \leq R$ is certainly bounded. Furthermore, the eigenvalues (40) can be divergent on the ring, but with a positive divergent part. Moreover, the acausality of the chosen seed metric does not imply that conditions (36) cannot be satisfied. Finally, the hydrostatic pressure must be vanishing at the boundary $r=R$. As stated above, expressions (39) and (40) are too long and complicated to easily find the correct expressions for $B\left(r, \theta, a^{2}\right)$ and $H\left(r, \theta, a^{2}\right)$ (if they exist!).

\section{Example Two: Conformally Flat Anisotropic Seed Metric}

In this last section we give an example of a class of Kerr interior solutions with a more appealing geometry than the one considered above, i.e. with boundary surfaces and therefore without distributional source at $r=R$. To this purpose, we start with the class of static anisotropic conformally flat solutions found by Stewart (see [14]). The starting point is the energymomentum tensor $T_{\mu \nu}$ in the form (see [21]):

$$
T_{\mu \nu}=(\epsilon+p) u_{\mu} u_{\nu}+p g_{\mu \nu}+(\sigma-p) \xi_{\mu} \xi_{\nu}
$$

where $u_{\mu}$ is the anisotropic fluid four-velocity, $\xi_{\mu}$ is a spacelike unit fourvector pointing in the direction of the anisotropy, $\epsilon$ is the rest energy density, $\sigma$ is the pressure along the anisotropy and $p$ is the pressure on a plane perpendicular to the anisotropy direction.

A class of static solutions, matching at the boundary with the vacuum Schwarzschild metric, can be obtained with:

$$
\epsilon=\frac{6 m}{R^{3}\left(1-\frac{3}{5} q\right)}\left(1-\frac{q r^{2}}{R^{2}}\right) \quad, \quad 0 \leq q \leq 1
$$

For $q=0$ we ragain the incompressible Schwarzschild solution. As we have seen in section 2 , to obtain solutions with boundary surfaces, we need a 
$C^{1}$ expression for $e^{2 \mu(r)}$. This can be obtained if and only if we set $q=1$ $(\epsilon(R)=0)$. Consequently, the solution $(q=1)$, with (1), is:

$$
\begin{aligned}
& e^{2 \nu(r)}=e^{2 \Phi(r)}\left(c r^{2} e^{-2 \Phi(r)}+b\right)^{2}, \\
& e^{2 \mu(r)}=\frac{R^{3}}{\left(R^{3}-5 m r^{2}+\frac{3 m r^{4}}{R^{2}}\right)}, \\
& e^{2 \Phi(r)}=\frac{1}{2}\left[1+\sqrt{1-\frac{5 m r^{2}}{R^{3}}+\frac{3 m r^{4}}{R^{5}}}-\frac{5 m r^{2}}{2 R^{3}}\right], \\
& c=\frac{1}{2} \frac{e^{\Phi(R)}}{R^{2}}\left[\frac{3 m}{R}-1+\sqrt{1-\frac{2 m}{R}}\right], \\
& b=\frac{1}{2} e^{-\Phi(R)}\left[1-\frac{3 m}{R}+\sqrt{1-\frac{2 m}{R}}\right] .
\end{aligned}
$$

Note that both the metric metric functions $g_{t t}$ and $g_{r r}$ are $C^{1}$ on the boundary at $r=R$. Therefore, with the help of the procedure discussed above, we can write a class of interior Kerr solutions satisfying the conditions (12)-(15), i.e. the continuity of the first and the second fundamental form (boundary surfaces). The solutions so obtained are:

$$
\begin{aligned}
& e^{2 \mu_{-}}=\frac{\left[R^{3}+R a^{2} \cos ^{2} \theta\right] e^{A\left(a^{2}, r, \theta\right)}}{\left(R^{3}+R a^{2} \cos ^{2} \theta-5 m r^{2}+\frac{3 m r^{4}}{R^{2}}\right)}+S_{A}\left(a^{2}, r, \theta\right), \\
& e^{2 \Phi\left(a^{2}, r, \theta\right)}=\frac{1}{2}\left[1-\frac{5 m r^{2}}{2 R\left(R^{2}+a^{2} \cos ^{2} \theta\right)}\right. \\
& +\sqrt{\left.1-\frac{5 m r^{2}}{R\left(R^{2}+a^{2} \cos ^{2} \theta\right)}+\frac{3 m r^{4}}{R^{3}\left(R^{2}+a^{2} \cos ^{2} \theta\right)}\right]} \\
& e^{2 \nu_{-}}=e^{2 \Phi\left(a^{2}, r, \theta\right)\left[c r^{2} e^{-2 \Phi\left(a^{2}, r, \theta\right)}+b\right]^{2} e^{B\left(a^{2}, r, \theta\right)}+H_{B}\left(a^{2}, r, \theta\right)}, \\
& c=\frac{1}{2 R^{2}} e^{\Phi\left(a^{2}, R, \theta\right)}\left[\frac{3 m R}{R^{2}+a^{2} \cos ^{2} \theta}-1+\sqrt{1-\frac{2 m R}{R^{2}+a^{2} \cos ^{2} \theta}}\right], \\
& b=\frac{1}{2} e^{-\Phi\left(a^{2}, R, \theta\right)}\left[1-\frac{3 m R}{R^{2}+a^{2} \cos ^{2} \theta}+\sqrt{1-\frac{2 m R}{R^{2}+a^{2} \cos ^{2} \theta}}\right],
\end{aligned}
$$

where $A\left(a^{2}, r, \theta\right)$ and $B\left(a^{2}, r, \theta\right)$ are arbitrary regular functions such that:

$$
A(0, r, \theta)=A\left(a^{2}, R, \theta\right)=B(0, r, \theta)=B\left(a^{2}, R, \theta\right)=0
$$


and (see discussion before equation (26) $) S_{A}$ and $H_{B}$ are regular vanishing functions for $a=0$ and $r=R$ and depend on the choice made for $A$ and $B$. Setting regular expressions for $A$ and $B$, the metric so obtained is regular for $R>\frac{25}{12} m$ and on the symmetry axis, with the exception of the ring singularity on the equatorial plane for $r=0, \theta=\frac{\pi}{2}$.

Also in this case, since the expressions involved are very long and show a complicated dependence on $A, S_{A}, B, H_{B}$, the study of energy conditions is not a simple task. However, the energy-momentum for (47) is again of type $\mathrm{I}$, and the reasonings made in section 5 are again valid.

As a final remark, we point out that the procedure discussed in this paper can be applied not only for obtaining Kerr interior solutions but also to find interior solutions matching with a general asymptotically flat vacuum stationary spacetime, provided that we start with a known reasonable static metric.

\section{References}

[1] R. P. Kerr, Phys. Rev. Lett. 11, 237 (1963).

[2] P. Florides, Nuovo Cimento B 13, 1 (1973).

[3] P. Florides and J. Synge, Proc. Roy. Soc. A 280, 459 (1964).

[4] M. Gurses and F. Gursey, J. Math Phys. 16, 2385 (1975).

[5] H. Wahlquist, Phys. Rev. 172, 5, 1291 (1968).

[6] L. Herrera and L. Jimenez, J. Math Phys. 23(12), 2339 (1982).

[7] E. T. Newman and A. Janis, J. Math. Phys. 6, 915 (1965).

[8] R. H. Boyer and T. G. Price, Proc. Camb. Phil. Soc. 61, 531 (1965).

[9] W. Israel, Nuovo Cimento 44, 1 (1966).

[10] W. Israel, Phys. Rev. D 2, 641 (1970).

[11] S. P. Drake and R. Turolla, Class. Quantum Grav. 14, 1883 (1997).

[12] S. P. Drake and Szekeres, Gen. Rel. Grav. 32, 445 (2000) (Preprint gr-qc/9807001).

[13] J. R. Oppenheimer and G. Volkov, Phys. Rev. 55, 374 (1939). 
[14] B. W. Stewart, J. Phys. A: Math Gen 15, 2419 (1982).

[15] R. Boyer and R. W. Lindquist, J. Math. Phys. 8, 265 (1967).

[16] H. Bondi, Proc. R. Soc. A 282, 303 (1964).

[17] S. W. Hawking and G. F. R. Ellis, The Large Scale strutcure of spacetime (Cambridge: Cambridge University Press, 1973).

[18] H. A. Buchdahl, Phys. Rev. 116, 1027 (1959).

[19] T. Papakostas, Int. J. Mod. Phys. D 10, 869 (2001).

[20] J. B. Hartle, Astrophys. J. 150, 1005 (1967).

[21] P. S. Letelier, Phys. Rev. D 22, 807 (1980). 